\title{
Vitali's theorem in vector lattices
}

Dedicated to Professor Yoshie Katsurada on her 60th birthday

$$
\text { By Shozo KosHi }
$$

\section{§1. Introduction}

Let $\Omega$ be a measure space with finite measure $\mu$. Then, Vitali's theorem announces : let $f_{n}(n=1,2, \cdots)$ be a sequence of summable functions on $\Omega$ with equi-absolutely continuous integrals and $f_{n}$ converges to $f$ in measure. Then,

$$
\lim _{n \rightarrow \infty} \int_{\Omega} f_{n} d \mu=\int_{\Omega} f d \mu .
$$

In this note, we shall generalize the Vitali's theorem to vector lattices.

\section{§ 2. Convergences in vector lattices.}

Let $R$ be a $\sigma$-complete vector lattice i. e. $\bigcap_{n=1}^{\infty} a_{n}$ exists for every positive elements $0 \leqq a_{n} \in R(n=1,2, \cdots)$. In the sequel, we assume that $R$ is $\sigma$ complete. For $a_{n} \in R(n=1,2, \cdots)$, if $\bigcap_{m=1}^{\infty}\left(\bigcup_{n \geq m} a_{n}\right)$ and $\bigcup_{m=1}^{\infty}\left(\bigcap_{n \geq m} a_{n}\right)$ exist and equal to $a$, then we denote that

$$
o-\lim _{n \rightarrow \infty} a_{n}=a .
$$

In this case, we say that the sequence $\left\{a_{n}\right\}$ is order-convergent to $a$. It is easy to see that $o$ - $\lim a_{n}=a$ iff there exist $b_{n} \downarrow 0$ (i. e. $b_{1} \geqq b_{2} \geqq \cdots$ with $\bigcap_{n=1}^{\infty} b_{n}=0$ ) such that

$$
\left|a_{n}-a\right| \leqq b_{n}
$$

where $|x|=x \cup(-x)=x^{+}+x^{-}$for $x \in R$.

We shall define star-order-convergence as follows: a sequence $a_{n}(n$ $=1,2, \cdots)$ is said to be star-order-convergent to $a$ if for every subsequence of $\left\{a_{n}\right\}$, there exists its subsequence which is order-convergent to $a$. We shall denote

$$
s-o-\lim a_{n}=a
$$

if $a_{n}(n=1,2, \cdots)$ is star-order-convergent to $a$.

For a subset $M$ of $R$, we denote $M^{\perp}=\{a:|a| \cap|b|=0$ for all $b \in M\}$. 
If we can decompose $a \in R$ into as follows:

$$
\text { (A) } \quad a=a_{1}+a_{2}
$$

with $a_{1} \in M$ and $a_{2} \in M^{\perp}$, then $M$ is called normal.

If $M$ is normal, then $M=M^{\perp \perp}$ and the decomposition $(A)$ of $a$ is uniquely determined. Namely,

$$
a=a_{1}+a_{2}, \quad a=a_{1}^{\prime}+a_{2}^{\prime}, \quad a_{1}, a_{1}^{\prime} \in M, \quad a_{2}, a_{2}^{\prime} \in M^{\perp}
$$

imply $\quad a_{1}=a_{1}^{\prime}$ and $a_{2}=a_{2}^{\prime}$.

We see that the operator $[M] a=a_{1}$ is linear and lattice-homomorphic. [M] is called a projection operator.

The normal subsets of $R$ (or equivalently projection operators) constitutes a Boolean lattice by the usual order.

Let $R$ be $\sigma$-complete. For $0 \leqq p \in R$, the subset $\{p\}^{\perp \perp}$ is normal and

$$
\left[\{p\}^{\perp \perp}\right] a=(\text { denoted by }[p] a)=\bigcup_{n=1}^{\infty}(n p \cap a) \text { for } a \geqq 0 \text {. }
$$

In general, $[p] a=[p] a^{+}-[p] a^{-}$.

$\{[N] ;[p] \geqq[N]\}$ is $\sigma$-complete as a Boolean lattice and for every [N] with $[p] \geqq[N]$ there exists $q \in R$ with $[N]=[q]$.

For arbitrary $p \in R,[p]=[|p|]$.

Let $\left[p_{n}\right]$ be a sequence of projection operators. $\left[P_{n}\right]$ is order-convergent to 0 if $\bigcap_{m=1}^{\infty}\left(\bigcup_{n \geqq m} P_{n}\right)=0$, i. e. there exists $\left[Q_{n}\right] \geqq\left[P_{n}\right]$ with $\left[Q_{1}\right] \geqq\left[Q_{2}\right] \geqq \cdots$ and $\bigcap_{n=1}^{\infty}\left[Q_{n}\right]=0$.

We shall write $\left[P_{n}\right] \downarrow 0$ if $\left[P_{1}\right] \geqq\left[P_{2}\right] \geqq \cdots$ and $\bigcap_{n=1}^{\infty}\left[P_{n}\right]=0$.

We shall denote $\left[P_{n}\right] \downarrow \downarrow 0$ if for every subsequence of $\left[P_{n}\right]$ there exists its subsequence order-convergent to 0 . lattice.

Now, we shall consider a special convergence in a $\sigma$-complete vector

We shall denote

$$
\circledast-\lim _{n \rightarrow \infty} a_{n}=a
$$

if there exists $\left[P_{m}\right] \downarrow \downarrow 0$ such that $\left(\mathrm{I}-\left[P_{m}\right]\right) a_{n}$ is star-order-convergent to $\left(\mathrm{I}-\left[P_{m}\right]\right) a(m=1,2, \cdots)$. In the case of $L_{1}$-space (the totality of summable functions) $f_{n} \rightarrow f$ (in measure) implies $f_{n} \rightarrow f$ (in above sense).

It is easy to see that if $\circledast-\lim _{n \rightarrow \infty} a_{n}=a$, then there exists $\left[p_{m}\right] \downarrow \downarrow 0$ such that $p_{m} \in R(m=1,2, \cdots)$ and $\left(\mathrm{I}-\left[p_{m}\right]\right) a_{n}$ is star-order-convergent to $\left(\mathrm{I}-\left[p_{m}\right]\right) a$ for all $m=1,2, \cdots$.

We see easily $\left[p_{n}\right] \downarrow 0$ and $\left[q_{n}\right] \downarrow 0$ imply $\left[p_{n}\right] \cup\left[q_{n}\right] \downarrow 0$. Hence, we see 
that if $\circledast-\lim _{n \rightarrow \infty} a_{n}=a$ and $\circledast-\lim _{n \rightarrow \infty} b_{n}=b$, then

$$
\begin{aligned}
& \circledast-\lim _{n \rightarrow \infty}\left(a_{n}+b_{n}\right)=\circledast-\lim _{n \rightarrow \infty} a_{n}+\circledast-\lim _{n \rightarrow \infty} b_{n}, \\
& \circledast-\lim _{n \rightarrow \infty}\left(a_{n} \cup b\right)=a \cup b, \circledast-\lim \left(a_{n} \cap b\right)=a \cap b, \\
& \circledast-\lim _{n \rightarrow \infty}\left|a_{n}-a\right|=0 .
\end{aligned}
$$

\section{§3. Equi-continuous subsets}

Let $a$ be a linear functional on $R . \quad a$ is said to be o-continuous linear functional if $o-\lim a_{n}=a$ implies $\bar{a}\left(a_{n}\right) \rightarrow \bar{a}(a)$.

It is easy to see that if $\bar{a}$ is an $o$-continuous linear functional on $R$, then

$$
\sup _{0 \leqq b \leqq|a|}|\bar{a}(b)|<+\infty \text { for all } a \in R .
$$

The totality of o-continuous linear functionals is denoted by $\bar{R}$ and is called an order-conjugate space of $R$. Since $R$ is reduced to 0 in some occasion, we assume that $R$ is not trivial in the sense that for every $a \neq 0$, there exists $a \in \bar{R}$ with $\bar{a}(a) \neq 0$.

$\bar{a} \geqq \bar{b}$ means that $\bar{a}(a) \geqq \bar{b}(a)$ for all $a \geqq 0$.

By this order, $\mathbb{R}$ is a complete vector lattice. In the sequel, we assume that $R$ is not trivial. $A$ subset $\Gamma$ of $R$ is equi-continuous if for $\bar{a}_{n} \downarrow 0, \bar{a}_{n} \in \bar{R}$ and $\varepsilon>0$, there exists a natural number $n_{0}$ with

$$
a_{n_{0}}(|a|) \leqq \varepsilon \text { for all } a \in \Gamma .
$$

By definition, if $\Gamma$ is an equi-continuous subset of $R$, then $N[\Gamma]=\{b$; $|b| \leqq|a|$ for some $a \in \Gamma\}$ is also an equi-continuous subset. It is known that $\Gamma$ is equi-continuous iff $\Gamma$ is relative compact by the weak topology induced by $\bar{R}$.

For $\left[P_{n}\right] \downarrow 0$ and $a \in R, a\left[P_{n}\right](a)=a\left(\left[P_{n}\right] a\right)$ is also o-continuous linear functional for all $n$ and $a\left[P_{n}\right] \downarrow 0$.

\section{§4. Vitali's theorem}

Vitali's theorem for summable functions can be formulated as follows in the case of vector lattices.

THEOREM 1. Let $a_{n} \in R(n=1,2, \cdots)$ be an equi-continuous sequences and $\circledast-\lim _{n \rightarrow \infty} a_{n}=a$. Then, $a_{n}$ is weakly convergent to $a\left(i . e . \bar{a}\left(a_{n}\right) \rightarrow \bar{a}(a)\right.$ for all $a \in \bar{R})$.

PROof. Let $\left[p_{n}\right] \downarrow \downarrow 0$ and $0 \leqq a \in \mathbb{R}$. We shall prove that we can find 
a natural number $n_{0}$ such that

$$
\bar{a}\left(\left[p_{n}\right]\left|a_{m}\right|\right) \leqslant \varepsilon \quad \text { for } n \geqq n_{0} \text { and } m=1,2, \cdots .
$$

If not, we find $\varepsilon>0$ and $m_{\nu} \leqq m_{\nu+1} \leqq \cdots$ and $n_{\nu} \leqq n_{\nu+1} \leqq \cdots$ such that

$$
\bar{a}\left(\left[p_{n_{\nu}}\right]\left|a_{m_{\nu}}\right|\right) \geqq \varepsilon \quad \nu=1,2, \cdots .
$$

By assumption, there exists a subsequence $\left[q_{\nu}\right]$ of $\left[p_{n_{\nu}}\right](\nu=1,2, \cdots)$ orderconvergent to 0 such that

$$
\bar{a}\left(\left[q_{\nu}\right]\left|a_{m_{\nu}}\right|\right) \geqq \varepsilon .
$$

This contradicts to the equi-continuity of $\Gamma=\left\{a_{n}\right\}$.

We shall prove that $s-0-\lim a_{n}=a$ implies $\bar{a}\left(a_{n}\right) \rightarrow \bar{a}(a)$.

If not, there exists $n_{\nu}(\nu=1,2, \cdots)$ such that

$$
\left|\bar{a}\left(a_{n_{\nu}}-a\right)\right| \geqq \varepsilon \text { for some } \varepsilon>0 .
$$

By assumption, we find a subsequence of $\left\{a_{n_{\nu}}\right\}$ order-convergent to $a$. This is a contradiction, since $a$ is continuous by order-convergence.

Let $\circledast-\lim _{n \rightarrow \infty} a_{n}=a$. There exists $[a] \geqq\left[p_{m}\right] \downarrow \downarrow 0$ such that

$$
s-o-\lim _{n \rightarrow \infty}\left(\mathrm{I}-\left[p_{m}\right]\right) a_{n}=\left(\mathrm{I}-\left[p_{m}\right]\right) \mathrm{a} .
$$

Hence, choosing $m$ such that $\mid \bar{a}\left(\left[p_{m}\right]\left(a_{n}-a\right) \mid<\varepsilon\right.$, we have

$$
\left|\bar{a}\left(a_{n}\right)-\bar{a}(a)\right| \leqq\left|\bar{a}\left(\left[p_{m}\right]\left(a_{n}-a\right)\right)\right|+\left|\bar{a}\left(\left(\mathrm{I}-\left[p_{m}\right]\right)\left(a_{n}-a\right)\right)\right| \leqq 2 \varepsilon
$$

for sufficient large $n$.

This proves Theorem 1.

COROLlary. Let $\circledast-\lim _{n \rightarrow \infty} a_{n}=a . \quad\left\{a_{n}\right\}$ is weakly convergent to $a$ if and only if $\left\{a_{n}\right\}$ is equi-continuous.

\section{§5. $|w|$-convergence.}

By definition,

$|w|-\lim _{n \rightarrow \infty} a_{n}=a$ iff $\lim _{n \rightarrow \infty} \bar{a}\left(\left|a_{n}-a\right|\right)=0$ for all $\bar{a} \in \bar{R} . \quad|w|-\lim _{n \rightarrow \infty} a_{n}=a$ implies that $a_{n}$ is weakly convergent to $a$. But, in general the converse is not true.

THEOREM 2. Under the assumption of Theorem 1, we have $|w|-\lim _{n \rightarrow \infty} a_{n}=a$.

PROOF. If $\left\{a_{n}\right\}$ is equi-continuous, then $\left\{x_{n}\right\}$ is equi-continuous where $x_{n}=\left|a_{n}-a\right|$. We see easily

$$
\circledast-\lim _{n \rightarrow \infty} a_{n}=a \text { iff } \circledast-\lim _{n \rightarrow \infty} x_{n}=\circledast-\lim _{n \rightarrow \infty}\left|a_{n}-a\right|=0 .
$$


By Theorem 1, $\left\{x_{n}\right\}$ is weakly convergent to 0 . But this means that

$$
|w|-\lim _{n \rightarrow \infty} a_{n}=a \text {. }
$$

Remark. If $R$ is a space of summable functions defined on a finite measure space, under the assumption of Theorem 1 , we have $\lim _{n \rightarrow \infty}\left\|a_{n}-a\right\|=0$ by Theorem 2 .

\section{Department of Mathematics Hakkaido University}

\section{References}

[1] H. NAKANo: Modulared semi-ordered linear spaces, Maruzen, Tokyo, 1950.

[2] I. NATANSON: Theory of functions of a real variable, (English translation) Ungar, New York, 1955.

(Received August 20, 1971) 